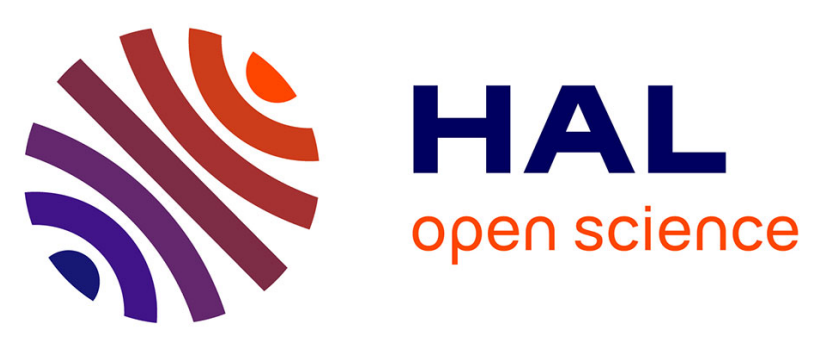

\title{
A Globally Exponentially Stable Speed Observer for a Class of Mechanical Systems: Experimental and Simulation Comparison with High-Gain and Sliding Mode Designs
}

Stanislav Aranovskiy, Romeo Ortega, Jose Guadalupe Romero, Dmitry

Sokolov

\section{To cite this version:}

Stanislav Aranovskiy, Romeo Ortega, Jose Guadalupe Romero, Dmitry Sokolov. A Globally Exponentially Stable Speed Observer for a Class of Mechanical Systems: Experimental and Simulation Comparison with High-Gain and Sliding Mode Designs. International Journal of Control, 2019, 92 (7), pp.1620-1633. 10.1080/00207179.2017.1404130 . hal-01658753

\section{HAL Id: hal-01658753 \\ https://hal.inria.fr/hal-01658753}

Submitted on 7 Dec 2017

HAL is a multi-disciplinary open access archive for the deposit and dissemination of scientific research documents, whether they are published or not. The documents may come from teaching and research institutions in France or abroad, or from public or private research centers.
L'archive ouverte pluridisciplinaire HAL, est destinée au dépôt et à la diffusion de documents scientifiques de niveau recherche, publiés ou non, émanant des établissements d'enseignement et de recherche français ou étrangers, des laboratoires publics ou privés. 


\title{
A Globally Exponentially Stable Speed Observer for a Class of Mechanical Systems: Experimental and Simulation Comparison with High-Gain and Sliding Mode Designs
}

\author{
S. Aranovskiya, ${ }^{\mathrm{a}, \mathrm{b}}$, R. Ortega ${ }^{\mathrm{c}}$, J. G. Romero ${ }^{\mathrm{d}}$, and D. Sokolove,f \\ ${ }^{a}$ Equipe Automatique, CentaleSupélec - IETR, Avenue de la Boulaie - CS 47601, 35576 \\ Cesson-Sévigné, France \\ ${ }^{\mathrm{b}}$ Department of Control Systems and Informatics, ITMO University, 197101 Saint \\ Petersburg, Russia \\ ${ }^{\mathrm{c}}$ Laboratoire des Signaux et Systèmes, CNRS-CentraleSupélec, Plateau du Moulon, 91192 \\ Gif-sur-Yvette, France \\ ${ }^{\mathrm{d}}$ Departamento Académico de Sistemas Digitales, Instituto Tecnológico Autónomo de \\ México-ITAM, 01080, Distrito Federal, México \\ eUniversité de Lorraine, LORIA, UMR 7503, Vandœuvre-lès-Nancy, F-54506, France \\ ${ }^{\mathrm{f}} \mathrm{CNRS}$, LORIA, UMR 7503, Vandouvre-lès-Nancy, F-54506, France
}

\section{ARTICLE HISTORY}

Compiled December 7, 2017

\begin{abstract}
It is shown in the paper that the problem of speed observation for mechanical systems that are partially linearisable via coordinate changes admits a very simple and robust (exponentially stable) solution with a Luenberger-like observer. This result should be contrasted with the very complicated observers based on immersion and invariance reported in the literature. A second contribution of the paper is to compare, via realistic simulations and highly detailed experiments, the performance of the proposed observer with well-known high-gain and sliding mode observers. In particular, to show that - due to their high sensitivity to noise, that is unavoidable in mechanical systems applications - the performance of the two latter designs is well below par.
\end{abstract}

\section{KEYWORDS}

Nonlinear systems, observer theory, mechanical systems, high-gain and sliding mode observers

\section{Introduction}

In this paper we are interested in the problem of speed observation of mechanical systems that are partially linearisable via coordinate changes (PLvCC). This class, formally defined in (Venkatraman, Ortega, Sarras, \& Van der Schaft, 2010), consists of mechanical systems whose dynamics becomes linear in velocity after a partial coordinate transformation, e.g., a linear transformation of the velocities. PLvCC mechanical systems have been extensively studied (Bedrossian \& Spong, 1995; Chang \& McLenaghan, 2013; Romero \& Ortega, 2015; Venkatraman et al., 2010) because, on 
one hand, observer design and controller synthesis are simplified for them while, on the other hand, there are many practical examples that satisfy this property. PLvCC mechanical systems have been characterized in (Venkatraman et al., 2010) via the solvability of a partial differential equation (PDE) defined by the inertia matrix. They contain as a particular case systems with zero Riemann symbols - also known as zero curvature systems - that are well known in analytical mechanics (Bedrossian \& Spong, 1995; Spivak, 1999). Some verifiable conditions of PLvCC have been recently reported in (Chang \& McLenaghan, 2013), where these systems are called quasi-linearizable.

In (Venkatraman et al., 2010) the problems of speed observation and position feedback stabilization of PLvCC systems are formulated and solved. The observer proposed in that paper is based on the immersion and invariance methodology proposed in (A. Astolfi, Karagiannis, \& Ortega, 2007), this leads to a complicated high-order design that includes a dynamic scaling factor that injects high-gain during the transients. The first main contribution of this paper is to show that an extremely simple Luenberger-like observer yields a globally exponentially stable (GES) solution to the speed observation problem of PLvCC systems. Moreover, a standard, quadratic, strict Lyapunov function to prove GES is constructed with classical "addition of cross terms" techniques (Khalil, 2002; Malisoff \& Mazenc, 2009). It should be underscored that the exponential qualifier is necessary to ensure that it can be combined-in a certainty equivalent way - with a full state-feedback controller to ensure a globally asymptotically stable (GAS) solution of the position feedback stabilization problem. In this respect, using the observer proposed here yields a simpler solution to the stabilization problem than the one given in (Venkatraman et al., 2010), but the details are omitted for brevity. Furthermore, as is amply discussed in (Khalil, 2002; Malisoff \& Mazenc, 2009), GES systems with strict Lyapunov functions enjoy strong robustness properties that cannot be ensured in the absence of such a function. Indeed, it is often the case that invoking arguments - that do not rely on the availability of Lyapunov functions - it is possible to prove global exponential convergence of the system but concluding some robustness properties for them is usually daunting task, see Proposition 9 of (Venkatraman et al., 2010) for a case in point.

As it is well known, it is undesirable to inject high-gain in a control loop. One of the deleterious effects of high-gain is the amplification of noise, which is unavoidable in any practical application, in particular, in mechanical systems. A second contribution of the paper is to show, via realistic simulations and highly detailed experiments, that the high sensitivity to noise of high-gain (Esfandiari \& Khalil, 1992; Khalil \& Praly, 2014) and sliding mode observers (Davila, Fridman, \& Levant, 2005) makes them less suitable for speed estimation of mechanical systems than the proposed one, which does not inject high-gain.

The remainder of the paper is structured as follows. In Section 2 we formulate the speed observation problem whose solution is given in Sections 3 and 4. Section 5 presents some simulation evidence of the proposed observer and two high-gain designs applied to the cart-and-pendulum system and the robotic leg, while Section 6 presents some compelling experimental evidence. We wrap-up the paper with concluding remarks in Section 7.

Notation. $I_{n}$ is the $n \times n$ identity matrix and $0_{n \times s}$ is an $n \times s$ matrix of zeros. For a vector $x \in \mathbb{R}^{n}$ and a square, symmetric matrix $A \in \mathbb{R}^{n \times n}$, we denote $|x|^{2}:=x^{\top} x$ and $\|A\|, \lambda_{m}\{A\}$ and $\lambda_{M}\{A\}$ the induced 2-norm and the minimum and maximum eigenvalue, respectively. All mappings are assumed smooth. Given a function $f: \mathbb{R}^{n} \rightarrow$ 
$\mathbb{R}$ we define $\nabla f:=\left(\frac{\partial f}{\partial x}\right)^{\top}$.

\section{Problem Formulation}

\subsection{The class of mechanical systems}

We consider in the paper mechanical systems whose dynamics is described by the Hamiltonian equations of motion

$$
\left[\begin{array}{c}
\dot{q} \\
\dot{\mathbf{p}}
\end{array}\right]=\left[\begin{array}{cc}
0_{n \times n} & I_{n} \\
-I_{n} & 0_{n \times n}
\end{array}\right] \nabla H(q, \mathbf{p})+\left[\begin{array}{c}
0_{n \times m} \\
G(q)
\end{array}\right] u,
$$

with total energy function $H: \mathbb{R}^{n} \times \mathbb{R}^{n} \rightarrow \mathbb{R}$

$$
H(q, \mathbf{p})=\frac{1}{2} \mathbf{p}^{\top} M^{-1}(q) \mathbf{p}+V(q),
$$

where $q, \mathbf{p} \in \mathbb{R}^{n}$ are the generalized positions and momenta, respectively, $u \in \mathbb{R}^{m}$ is the control input, $n \geq m$, the inertia matrix $M: \mathbb{R}^{n} \rightarrow \mathbb{R}^{n \times n}$ verifies $M(q)>0$, $V: \mathbb{R}^{n} \rightarrow \mathbb{R}$ is the potential energy function and $G: \mathbb{R}^{n} \rightarrow \mathbb{R}^{n \times m}$ is the full-rank input matrix.

The definition below identifies the class of mechanical systems that we consider in the paper.

Definition 2.1. (Venkatraman et al., 2010) The mechanical system (1) is said to be PLvCC if there exists a full rank mapping $\Psi: \mathbb{R}^{n} \rightarrow \mathbb{R}^{n \times n}$ such that the (partial) change of coordinates

$$
(q, p) \mapsto\left(q, \Psi^{\top}(q) \mathbf{p}\right)
$$

transforms (1) into

$$
\begin{aligned}
\dot{q} & =\mathcal{M}(q) p \\
\dot{p} & =-\Psi^{\top}(q)[\nabla V(q)-G(q) u]
\end{aligned}
$$

where, to simplify the notation, we have introduced the full-rank mapping $\mathcal{M}: \mathbb{R}^{n} \rightarrow$ $\mathbb{R}^{n \times n}$ as

$$
\mathcal{M}(q):=M^{-1}(q) \Psi^{-\top}(q) .
$$

Remark 1. As shown in (Venkatraman et al., 2010) the change of coordinates (2) transforms (1) into

$$
\left[\begin{array}{c}
\dot{q} \\
\dot{p}
\end{array}\right]=\left[\begin{array}{cc}
0_{n \times n} & \Psi(q) \\
-\Psi^{\top}(q) & J(q, p)
\end{array}\right] \nabla \bar{H}(q, p)+\left[\begin{array}{c}
0 \\
\Psi^{\top}(q) G(q)
\end{array}\right] u
$$

with new Hamiltonian $\bar{H}: \mathbb{R}^{n} \times \mathbb{R}^{n} \rightarrow \mathbb{R}$

$$
\bar{H}(q, p)=\frac{1}{2} p^{\top}\left[\Psi^{\top}(q) M(q) \Psi(q)\right]^{-1} p+V(q),
$$


and the $j k$-th element of the skew-symmetric matrix $J: \mathbb{R}^{n} \times \mathbb{R}^{n} \rightarrow \mathbb{R}^{n \times n}$ given by

$$
(J(q, p))_{j k}=-p^{\top}\left[(\Psi(q))_{j},(\Psi(q))_{k}\right], j, k \in \bar{n}
$$

with $[\cdot, \cdot]$ the standard Lie bracket (Spivak, 1999) and $(\cdot)_{j}$ the $j$-th column. In (Venkatraman et al., 2010) it is shown that (4) reduces to (3) if and only if

$$
\Psi^{\top}(q) \frac{\partial}{\partial q}\left\{\frac{1}{2} \mathbf{p}^{\top} M^{-1}(q) \mathbf{p}\right\}-\dot{\Psi}^{\top}(q) \mathbf{p} \equiv 0 .
$$

Interestingly, the latter is true if and only if a PDE in $\Psi(q)$, which is univocally defined by $M(q)$, admits a solution - see Assumption 1 in (Venkatraman et al., 2010). See also (Chang \& McLenaghan, 2013) for a geometric characterisation of the PLvCC property.

Remark 2. The main feature of PLvCC systems is that, as seen from (3), their dynamics is linear in momenta. Adopting a Lagrangian description of the system this means that in PLvCC systems the quadratic terms in velocity - appearing in the Coriolis and centrifugal forces vector - vanish when the dynamics is expressed in the new coordinates.

\subsection{Exponentially stable observer design problem}

Before presenting the observation problem that is formulated, and solved, in this paper we state the following assumption, which is standard in (open loop) observer design problems.

Assumption 2.2. $u \in \mathcal{L}_{\infty}$ and is such that $q, p \in \mathcal{L}_{\infty}$, with a known bound

$$
\max \left\{\|q\|_{\infty},\|p\|_{\infty}\right\} \leq K,
$$

where $\|\cdot\|_{\infty}$ is the $\mathcal{L}_{\infty}$ norm.

Momenta observation problem. Consider the mechanical system (3). Find two mappings

$$
F, H: \mathbb{R}^{n} \times \mathbb{R}^{n} \times \mathbb{R}^{n} \times \mathbb{R}^{m} \rightarrow \mathbb{R}^{n},
$$

such that (3) together with

$$
\begin{aligned}
\dot{\hat{q}} & =F(\hat{q}, \hat{p}, q, u) \\
\dot{\hat{p}} & =H(\hat{q}, \hat{p}, q, u),
\end{aligned}
$$

generates an error system

$$
\dot{e}=B(e, t),
$$

with

$$
e:=\operatorname{col}(\hat{q}-q, \hat{p}-p)=\operatorname{col}(\tilde{q}, \tilde{p}) \in \mathbb{R}^{2 n},
$$


the error coordinates, for which there exists a quadratic function $W: \mathbb{R}_{+} \times \mathbb{R}^{2 n} \rightarrow \mathbb{R}_{+}$,

$$
W(t, e):=\frac{1}{2} e^{\top} P(t) e
$$

with $P: \mathbb{R}_{+} \rightarrow \mathbb{R}^{n \times n}$ verifying

$$
c_{2} I_{2 n} \leq P(t) \leq c_{3} I_{2 n}
$$

and

$$
\dot{W} \leq-c_{4}|e|^{2},
$$

for some positive constants $c_{i}, i=2,3,4$. As is well-known (Khalil, 2002) these properties ensure GES of the zero equilibrium of (6). In particular, we have

$$
\left|\left[\begin{array}{c}
\tilde{q}(t) \\
\tilde{p}(t)
\end{array}\right]\right|^{2}=\frac{c_{3}}{c_{2}} e^{-\frac{2 c_{4}}{c_{3}} t}\left|\left[\begin{array}{c}
\tilde{q}(0) \\
\tilde{p}(0)
\end{array}\right]\right|^{2},
$$

for all $(\tilde{q}(0), \tilde{p}(0)) \in \mathbb{R}^{n} \times \mathbb{R}^{n}$ and all $t \geq 0$.

Remark 3. In the problem formulation above we are aiming at an exponentially convergent momenta observer with a strict Lyapunov function. As discussed in the introduction the exponential requirement is necessary to, invoking Proposition 9 of (Venkatraman et al., 2010), be able to use the observer to give a GAS solution to the position feedback regulation problem. On the other hand, the importance for robustness analysis of disposing of a strict Lyapunov function can hardly be overestimated- (Malisoff \& Mazenc, 2009, see) for a detailed discussion on this matter.

Remark 4. Notice that the system (3) does not verify the conditions for application of the classical "linearization up to an output injection" observers of (Krener \& Respondek, 1985). On the other hand, the observer proposed in (Venkatraman et al., 2010) - besides being extremely complex for practical application - does not satisfy the strict Lyapunov requirement and only global (exp.) convergence is established.

\section{Proposed Observer and Literature Review}

\subsection{A Luenberger observer and resulting error equation}

The system (3) suggests the following standard Luenberger observer

$$
\begin{aligned}
& \dot{\hat{q}}=\mathcal{M}(q) \hat{p}-L(\hat{q}-q) \\
& \dot{\hat{p}}=-\Psi^{\top}(q)[\nabla V(q)-G(q) u]-\Gamma \mathcal{M}^{\top}(q)(\hat{q}-q),
\end{aligned}
$$

where $L, \Gamma \in \mathbb{R}^{n \times n}, L, \Gamma>0$. The error equations take the familiar form,

$$
\begin{aligned}
& \dot{\tilde{q}}=-L \tilde{q}+\mathcal{M}(q) \tilde{p} \\
& \dot{\tilde{p}}=-\Gamma \mathcal{M}^{\top}(q) \tilde{q} .
\end{aligned}
$$


In the $e$ coordinates (7) the error equations (12) may be written as a linear timevarying (LTV) system of the form

$$
\dot{e}=\left[\begin{array}{cc}
-L & \mathcal{M}(q(t)) \\
-\Gamma \mathcal{M}^{\top}(q(t)) & 0_{n \times n}
\end{array}\right] e=: A(t) e,
$$

where, in view of Assumption 2.2, $A(t)$ and $\dot{A}(t)$ are bounded matrices. This kind of equations have been exhaustively studied in several contexts in the control literature, in particular, for adaptive systems (Anderson et al., 1986; Ioannou \& Sun, 2012; Marino \& Tomei, 1996; Sastry \& Bodson, 2011). It is well-known that a necessary and sufficient condition for GES is that the matrix $\mathcal{M}(q(t))$ - not necessarily square nor full-rankverifies a persistency of excitation (PE) condition. Namely, that there exists positive constants $T$ and $\epsilon$ such that

$$
\int_{t}^{t+T} \mathcal{M}^{\top}(q(s)) \mathcal{M}(q(s)) d s \geq \epsilon I_{n} .
$$

Notice that, in our case, this condition is clearly satisfied because $\mathcal{M}(q)$ is square and full-rank, therefore, $\mathcal{M}^{\top}(q) \mathcal{M}(q)$ is positive definite.

\subsection{Review of existing analysis results}

Unfortunately, as we discuss now, most proofs of GES of (12) available in the literature do not match the requirements of our problem formulation. In (Anderson et al., 1986; Ioannou \& Sun, 2012; Sastry \& Bodson, 2011) the equivalence between the PE condition (14) and GES of (13) is established without a strict Lyapunov function, but invoking instead properties of uniform complete observability of LTV systemsa feature that is ensured by the PE condition. The resulting proof is very long and technically involved and does not give much insight into the role of the various free parameters of the system, see (Loria, 2004; Panteley \& Loria, 1998) for some discussion and (Maghenem \& Loria, 2016; ?) for recent related developments. Although the existence of a strict Lyapunov function can be established there is no explicit expression for it, instead it is is given in terms of the integral along trajectories of the fundamental matrix.

In Lemma B.2.3 of (Marino \& Tomei, 1996) it is claimed that it is possible to prove that PE implies GES without invoking observability concepts. Unfortunately, the proof of this claim is wrong. Indeed, the derivation of the key inequality (B.43) relies on the following implication

$$
\Omega \in P E \Rightarrow \int_{t}^{t+T} z^{\top}(s) \Omega(s) \Omega^{\top}(s) z(s) d s \geq \epsilon \delta^{2}, \forall|z| \geq \delta
$$

where $\Omega, z \in \mathbb{R}^{n}$. The implication is clearly wrong because we cannot rule out the vector $z$ orthogonal (or converging to orthogonal) to $\Omega$. In Lemma A.3 of (?) a strict Lyapunov function is proposed but, similarly to the observability-based proofs mentioned above, requires an integration along trajectories for its construction.

Similarly, it is shown in Proposition 4 of (Barabanov \& Ortega, 2017) that Theorem 2 of the highly cited paper (Morgan \& Narendra, 1977) is also wrong, with a gap in 
the proof appearing in item d) of page $21 .^{1}$

In view of the situation described above, in the next section we give an alternative proof of GES of (13) via the construction of a strict Lyapunov function, which follows closely (Malisoff \& Mazenc, 2009). In spite of the simplicity of the construction, it seems that it has not been reported, neither in the control, nor in the robotics, literature.

\section{Global Exponential Stability Proof}

To streamline the presentation of the result we find convenient to define the following positive constants

$$
\begin{gathered}
k_{1}:=\max _{t \geq 0}\|\mathcal{M}(q(t))\|, k_{2}:=\max _{t \geq 0}\|\dot{\mathcal{M}}(q(t))\|, k_{3}:=\|L\|, k_{4}:=\lambda_{m}\{L\}, \\
k_{5}:=\|\Gamma\|, k_{6}:=\min \left\{1, \lambda_{m}\{\Gamma\}\right\}, k_{7}:=\max \left\{1, \lambda_{M}\{\Gamma\}\right\}
\end{gathered}
$$

Notice that, under Assumption 2.2, these constants are well defined and can be computed from (5). We also introduce two positive constants

$$
d_{1}:=k_{1} k_{3}+k_{2}, d_{2}:=\frac{1}{2 k_{1}^{2}}\left(k_{1} k_{3}+k_{2}\right)^{2}+k_{1}^{2} k_{5}+\frac{k_{4}}{2} .
$$

Proposition 4.1. The zero equilibrium of the error system (13) is GES with a strict Lyapunov function (8) verifying (9) and (10) where

$$
P(t):=\left[\begin{array}{cc}
c_{1} I_{n} & -\mathcal{M}(q(t)) \\
-\mathcal{M}^{\top}(q(t)) & c_{1} \Gamma^{-1}
\end{array}\right]
$$

and

$$
c_{1}:=\frac{2}{k_{4}} d_{2}+k_{1} k_{7}, c_{2}:=\frac{1}{k_{7}}, c_{3}:=\frac{1}{k_{6}}\left(\frac{2}{k_{4}} d_{2}+2 k_{1} k_{7}\right), c_{4}:=\min \left\{\frac{k_{1}^{2}}{2}, d_{2}+k_{1} k_{4} k_{7}\right\} .
$$

Proof. The gist of the proof is to show that (8), (15) verifies (9) and (10). Towards this end, first, define the quadratic function $E: \mathbb{R}^{2 n} \rightarrow \mathbb{R}_{+}$

$$
E(e):=\frac{1}{2} e^{\top}\left[\begin{array}{cc}
I_{n} & 0_{n \times n} \\
0_{n \times n} & \Gamma^{-1}
\end{array}\right] e .
$$

whose time derivative along (13) verifies

$$
\dot{E}=-\tilde{q}^{\top} L \tilde{q} \leq-k_{4}|\tilde{q}|^{2} .
$$

Notice also that

$$
k_{6} E(e) \leq \frac{1}{2}|e|^{2} \leq k_{7} E(e)
$$

\footnotetext{
${ }^{1}$ Theorem 2 of (Morgan \& Narendra, 1977) claims GAS in the simpler case when $A(t)$ is a rank one matrix.
} 
where we have used the inequalities

$$
\frac{1}{\lambda_{M}\{\Gamma\}}=\lambda_{m}\left\{\Gamma^{-1}\right\} \leq \lambda_{M}\left\{\Gamma^{-1}\right\}=\frac{1}{\lambda_{m}\{\Gamma\}} .
$$

Following (Malisoff \& Mazenc, 2009) we propose a Lyapunov function candidate

$$
W(t, e)=c_{1} E(e)+U(t, e)
$$

where $E(e)$ is defined in (16) and we defined the cross term function $U: \mathbb{R}_{+} \times \mathbb{R}^{2 n} \rightarrow \mathbb{R}$

$$
U(t, e):=-\tilde{q}^{\top} \mathcal{M}(q(t)) \tilde{p} .
$$

First, we compute the bounds (9). Towards this end, we have that

$$
|U(t, e)| \leq k_{1}|\tilde{q}||\tilde{p}| \leq k_{1} k_{7} E(e),
$$

consequently

$$
k_{1} k_{7} E(e)+U(t, e) \geq 0
$$

From (19) we get

$$
W(t, e)=\left(\frac{2}{k_{4}} d_{2}+k_{1} k_{7}\right) E(e)+U(t, e) \geq \frac{2}{k_{4}} d_{2} E(e) \geq E(e) \geq \frac{1}{2 k_{7}}|e|^{2},
$$

where, to obtain the second inequality, we used the fact that $\frac{2}{k_{4}} d_{2}>1$ and the last one follows from (18)-proving the lower bound of (9). From (19) the following upper bound for $W(t, e)$ can be established:

$$
W(t, e) \leq\left(\frac{2}{k_{4}} d_{2}+k_{1} k_{7}\right) E(e)+k_{1} k_{7} E(e)=c_{3} k_{6} E(e) \leq \frac{c_{3}}{2}|e|^{2} .
$$

To complete the proof we establish now the upper bound (10). The time derivative of $U(t, e)$ is given by

$$
\dot{U}=-\tilde{p}^{\top} \mathcal{M}^{\top}(q)[-L \tilde{q}+\mathcal{M}(q) \tilde{p}]-\tilde{p}^{\top} \dot{\mathcal{M}}^{\top}(q) \tilde{q}+\tilde{q}^{\top} \mathcal{M}(q) \Gamma \mathcal{M}^{\top}(q) \tilde{q},
$$

whose terms can be bounded as follows:

$$
\begin{aligned}
& \tilde{q}^{\top} \mathcal{M}(q) \Gamma \mathcal{M}^{\top}(q) \tilde{q} \leq k_{1}^{2} k_{5}|\tilde{q}|^{2}, \\
& \tilde{p}^{\top}\left(\mathcal{M}^{\top}(q) L-\dot{\mathcal{M}}^{\top}(q)\right) \tilde{q} \leq d_{1}|\tilde{q}||\tilde{p}|, \\
&-\tilde{p}^{\top} \mathcal{M}^{\top}(q) \mathcal{M}(q) \tilde{p} \leq-k_{1}^{2}|\tilde{p}|^{2} .
\end{aligned}
$$

This leads to

$$
\dot{U} \leq-k_{1}^{2}|\tilde{p}|^{2}+k_{1}^{2} k_{5}|\tilde{q}|^{2}+d_{1}|\tilde{q}||\tilde{p}|
$$


Using the triangle inequality

$$
d_{1}|\tilde{q}||\tilde{p}| \leq \frac{1}{2 k_{1}^{2}} d_{1}^{2}|\tilde{q}|^{2}+\frac{k_{1}^{2}}{2}|\tilde{p}|^{2}
$$

and the definition of $d_{2}$ one obtains

$$
\dot{U} \leq-\frac{k_{1}^{2}}{2}|\tilde{p}|^{2}+d_{2}|\tilde{q}|^{2}
$$

Combining the latter bound with (17) we get

$$
\begin{aligned}
\dot{W} & \leq-\frac{k_{1}^{2}}{2}|\tilde{p}|^{2}+d_{2}|\tilde{q}|^{2}-k_{4}\left(\frac{2}{k_{4}} d_{2}+k_{1} k_{7}\right)|\tilde{q}|^{2} \\
& \leq-\frac{k_{1}^{2}}{2}|\tilde{p}|^{2}-\left(d_{2}+k_{1} k_{4} k_{7}\right)|\tilde{q}|^{2} \leq-c_{4}|e|^{2},
\end{aligned}
$$

which completes the proof.

Remark 5. In the proposed observer (11) it has been assumed that the matrix $L$ is positive definite. This assumption has been made to simplify the proof, from which it is clear that any Hurwitz matrix $L$ will ensure the GES property-replacing the first term of the Lyapunov function (16) by $\frac{1}{2} \tilde{q}^{\top} P_{0} \tilde{q}$, with $P_{0} \in \mathbb{R}^{2 n \times 2 n}$ the positive definite solution of the Lyapunov matrix equation $P_{0} L+L^{\top} P_{0}<0$.

Remark 6. The derivations above show that any system of the form

$$
\begin{aligned}
\dot{e}_{1} & =B\left(e_{1}\right) e_{2} \\
\dot{e}_{2} & =f_{2}\left(e_{1}, u\right) \\
y & =e_{1},
\end{aligned}
$$

with $e_{1} \in \mathbb{R}^{n_{1}}, e_{2} \in \mathbb{R}^{n_{2}}, u \in \mathbb{R}^{m}$ and $B: \mathbb{R}^{n_{1}} \rightarrow \mathbb{R}^{n_{1} \times n_{2}}, f_{2}: \mathbb{R}^{n_{1} \times m} \rightarrow \mathbb{R}^{n_{2}}$ smooth mappings, admit a Luenberger-like observer like the one proposed here. GAS is guaranteed for all values of $n_{1}, n_{2}>0$, but the proof of GES imposes the constraints $n_{1} \geq n_{2}$ and $B\left(e_{1}\right)$ full rank. The latter condition ensures the existence of the positive constants $k_{1}$ and $c_{4}$. Although GAS observers for systems which are linear in the unmeasurable states, like the one above, are available in the literature (A. Astolfi et al., 2007), the proposed observer is particularly attractive due to its simplicity and the fact that it ensures GES.

\section{Two Simulation Examples}

In this section we present simulations of three speed observers applied to two PLvCC mechanical systems in the presence of, practically unavoidable, measurement noise. Besides the proposed Luenberger observer - called in the sequel GES observer (GESO)we simulate a high-gain observer (HGO) and a sliding mode observer (SMO).

The first considered example is the cart-pendulum system, which is a well-known 2-dof mechanical example. For this example we simulate the HGO reported in (Lee, Mukherjee, \& Khalil, 2015), which is designed exactly for the cart-pendulum system, 


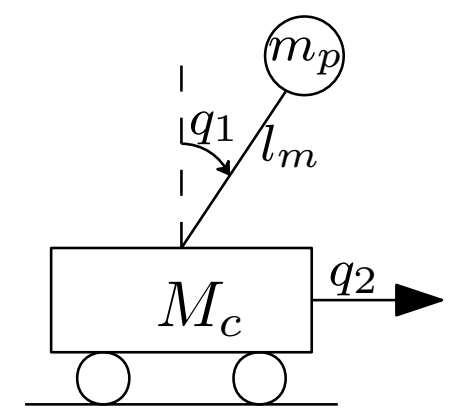

Figure 1. The inverted pendulum on a cart system

and the SMO reported in (Davila et al., 2005), which is designed for a pendulum and is modified in a straightforward manner to fit the cart-pendulum example.

The second example is the robotic leg, which is a 3-dof mechanical system. For this example we simulate the HGO and SMO reported in (Khalil \& Praly, 2014) and (Cruz-Zavala, Moreno, \& Fridman, 2010), respectively. These references present rather general theory of high-gain and sliding mode observers design and that be applied for the considered mechanical system.

Our interest in this section is twofold: first, to show the excellent behavior of the proposed GESO in spite of the presence of the noise. Second, to prove that - due to their high sensitivity to noise - the performances of the HGO and SMO are well below par.

\subsection{Cart-pendulum system}

The cart-pendulum system is a well-known example of a 2-dof, underactuation degree one, mechanical system depicted in Fig. 1 , where $M_{c}$ and $m_{p}$ are the masses of the cart and of the pendulum, respectively, and $l_{m}$ is the distance to the center of mass of the pendulum. Its dynamics, in a normalized form (Venkatraman et al., 2010), is described by (1) with

$$
\begin{aligned}
& M(q)=\left[\begin{array}{cc}
1 & b \cos \left(q_{1}\right) \\
b \cos \left(q_{1}\right) & m
\end{array}\right], \\
& V(q)=a \cos \left(q_{1}\right), G=\left[\begin{array}{l}
0 \\
1
\end{array}\right],
\end{aligned}
$$

where $a=g l_{m}^{-1}, b=l_{m}^{-1}$ and $m=\left(M_{c}+m_{p}\right)\left(m_{p} l_{m}^{2}\right)^{-1}$.

As shown in (Venkatraman et al., 2010) this system is PLvCC with $M^{-1}(q) \equiv$ $\Psi(q) \Psi^{\top}(q)$, therefore, it can be represented as (3) where

$$
\mathcal{M}(q)=\Psi(q):=\left[\begin{array}{cc}
\frac{\sqrt{m}}{\sqrt{m-b^{2} \cos ^{2}\left(q_{1}\right)}} & 0 \\
\frac{-b \cos \left(q_{1}\right)}{\sqrt{m} \sqrt{m-b^{2} \cos ^{2}\left(q_{1}\right)}} & \frac{1}{\sqrt{m}}
\end{array}\right] .
$$

The behaviour of the proposed GESO (11) is compared in simulations with the HGO and SMO proposed in (Lee et al., 2015) and (Davila et al., 2005), respectively. To design both observers we need the Coriolis matrix $C: \mathbb{R}^{2} \times \mathbb{R}^{2} \rightarrow \mathbb{R}^{2 \times 2}$, defined 
via the Christoffel symbols of the inertia matrix, which is given by

$$
C(q, \dot{q})=\left[\begin{array}{cc}
0 & 0 \\
-b \dot{q}_{1} \sin \left(q_{1}\right) & 0
\end{array}\right] .
$$

The HGO has the form

$$
\begin{aligned}
& \dot{\hat{x}}_{1}=\hat{x}_{2}+\left(\frac{h_{1}}{\varepsilon_{h}}\right) \tilde{x}_{1} \\
& \dot{\hat{x}}_{2}=M^{-1}\left(x_{1}\right)\left[G u-\nabla V\left(x_{1}\right)-C\left(x_{1}, \hat{x}_{2}\right) \hat{x}_{2}\right]+\left(\frac{h_{2}}{\varepsilon_{h}^{2}}\right) \tilde{x}_{1}
\end{aligned}
$$

where $x_{1}=q, x_{2}=\dot{q}$, its estimates are $\hat{x}_{1}$ and $\hat{x}_{2}$, respectively, the observation errors are defined as

$$
\tilde{x}_{1}=x_{1}-\hat{x}_{1}=: \operatorname{col}\left(\tilde{x}_{1_{1}}, \tilde{x}_{1_{2}}\right)
$$

and $h_{1}, h_{2}, \varepsilon_{h}$ are positive tuning parameters. The SMO is defined by the following equations

$$
\begin{aligned}
& \dot{\hat{x}}_{1}=\hat{x}_{2}+\Phi_{1}\left(\tilde{x}_{1}\right) \\
& \dot{\hat{x}}_{2}=M^{-1}\left(x_{1}\right)\left(G u-\nabla V\left(x_{1}\right)-C\left(x_{1}, \hat{x}_{2}\right) \hat{x}_{2}\right)+\Phi_{2}\left(\tilde{x}_{1}\right)
\end{aligned}
$$

where the expressions for $\Phi_{1}$ and $\Phi_{2}$ are given in (Davila et al., 2005) as

$$
\begin{gathered}
\Phi_{1}\left(\tilde{x}_{1}\right)=\left[\begin{array}{c}
1.5 \sqrt{\mu_{1}}\left|\tilde{x}_{1_{1}}\right|^{\frac{1}{2}} \operatorname{sign}\left(\tilde{x}_{1_{1}}\right) \\
1.5 \sqrt{\mu_{2}}\left|\tilde{x}_{1_{2}}\right| \frac{1}{2} \operatorname{sign}\left(\tilde{x}_{1_{2}}\right)
\end{array}\right], \\
\Phi_{2}\left(\tilde{x}_{1}\right)=\left[\begin{array}{l}
1.1 \mu_{1} \operatorname{sign}\left(\tilde{x}_{1_{1}}\right) \\
1.1 \mu_{2} \operatorname{sign}\left(\tilde{x}_{1_{2}}\right)
\end{array}\right],
\end{gathered}
$$

with positive gains $\mu_{1}$ and $\mu_{2}$. Note that since both SMO and GESO are given by four first-order differential equations, the HGO (21) is also chosen to be of order four. An extended HGO is further considered in Section 6.

For the numerical simulations we chose the system parameters $a=1, b=0.1$ and $m=1$. The simulation scenario for the three observers is $u(t) \equiv 0, q(0)=$ $\left[\begin{array}{ll}\frac{\pi}{2}-0.2,-0.1\end{array}\right]^{\top}, \dot{q}(0)=\left[\begin{array}{ll}0.4, & 0.35\end{array}\right]^{\top}$ and $\hat{q}(0)=q(0), \hat{\dot{q}}(0)=\hat{p}(0)=\left[\begin{array}{ll}0, & 0\end{array}\right]^{\top}$. The HGO (21) and the SMO (22) are tuned according to the recommendations given in (Lee et al., 2015) and (Davila et al., 2005), respectively, and are given as $h_{1}=3 \cdot 10^{-2}$, $h_{2}=2 \cdot 10^{-4}, \epsilon_{h}=0.01$ for the HGO, and $\mu_{1}=2.2, \mu_{2}=4$ for the SMO.

The gains of the proposed Luenberger observer (11) are chosen to have approximately the same transient time as SMO and HGO, yielding, $L=10 I_{2}$ and $\Gamma=70 I_{2}$. Note that the GESO estimates the generalized momenta vector $\hat{p}$, and the SMO and HGO estimate the velocity $\hat{\dot{q}}$, thus conversion of momenta to velocity is performed for comparison.

In an ideal case when both $q_{1}$ and $q_{2}$ are measured without any distortion, the three observers perform well, and simulation results for such a case are not of interest. Next, we consider the following realistic scenario of noisy measurements. 


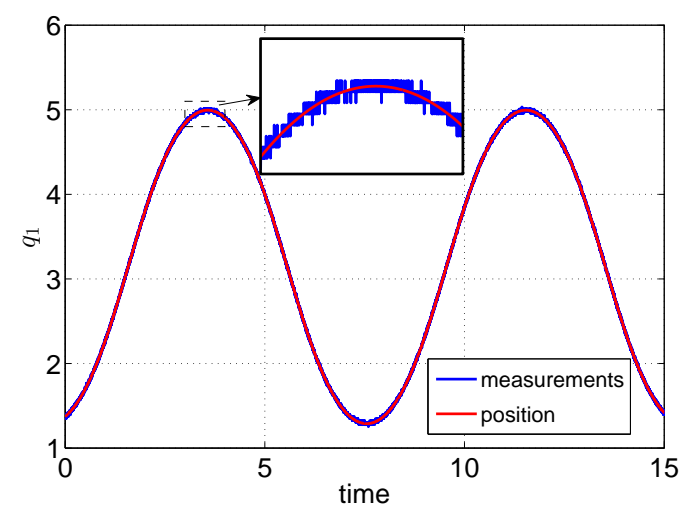

(a) Trajectory $q_{1}(t)$

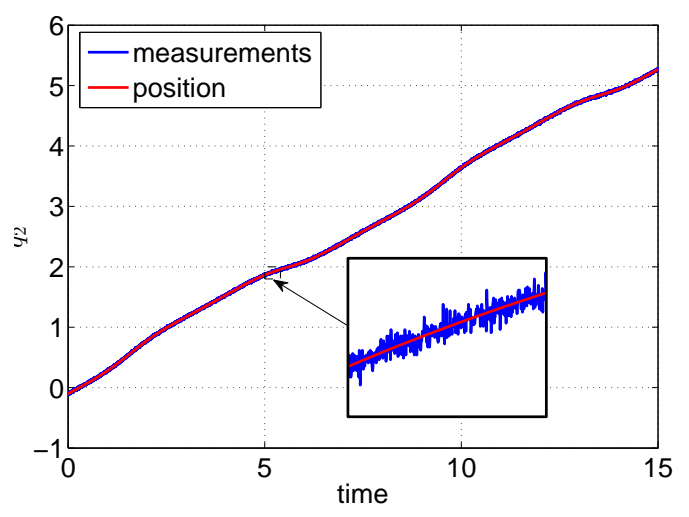

(b) Trajectory $q_{2}(t)$

Figure 2. Trajectories $q_{1}(t)$ and $q_{2}(t)$ in the considered operation scenario, real position and noisy measurements

Table 1. Numerical comparison of the observers.

\begin{tabular}{ccccc}
\hline & \multicolumn{2}{c}{$\dot{q}_{1}$} & \multicolumn{2}{c}{$\dot{q}_{2}$} \\
\cline { 2 - 5 } & $M E_{1} \cdot 10^{2}$ & $M S E_{1} \cdot 10^{4}$ & $M E_{2} \cdot 10^{2}$ & $M S E_{2} \cdot 10^{4}$ \\
\hline HGO & 3.1 & 15.5 & 2.6 & 10.5 \\
\hline SMO & 2.0 & 12.6 & 1.8 & 4.9 \\
\hline GESO & 0.5 & 0.3 & 0.4 & 0.2 \\
\hline
\end{tabular}

- Both $q_{1}$ and $q_{2}$ are perturbed by an additive normally (Gaussian) distributed random measurement noise with zero mean and a small variance $10^{-4}$.

- The noisy measurements are sampled with an analogue-to-digital converter with the quantization intervals $\frac{2 \pi}{256}$ for $q_{1}$ and $\frac{1}{500}$ for $q_{2}$.

- The sampling frequency is fixed as $1 \mathrm{kHz}$.

In Fig. 2 the trajectories $q_{1}(t)$ and $q_{2}(t)$ are given, note the relatively small distortion of the measurements. Estimates of the velocities $\dot{q}_{1}$ and $\dot{q}_{2}$ are given in Fig. 3, which clearly shows the superior performance of GESO. To quantify this fact a numerical comparison of the observers is given in Table 1, where the metrics

$$
\begin{aligned}
M E_{j} & :=\frac{1}{N} \sum_{i=1}^{N}\left|\hat{\dot{q}}_{j}\left(t_{i}\right)-\dot{q}_{j}\left(t_{i}\right)\right|, \\
M S E_{j} & :=\frac{1}{N} \sum_{i=1}^{N}\left(\hat{\dot{q}}_{j}\left(t_{i}\right)-\dot{q}_{j}\left(t_{i}\right)\right)^{2},
\end{aligned}
$$

are computed with $t_{i}, i=1, \ldots, N$, being the sampling instants, and $j=1,2$ for $q_{1}$ and $q_{2}$, respectively. To distinguish the effect of the noise with respect to the errors due to the mismatched initial conditions the metrics were computed omitting the transients. The experiment duration is 15 seconds, and dropping away the first 1.5 seconds of transients, the number of samples $N$ equals to 13500 . It can be seen that the proposed GESO significantly outperforms the HGO and the SMO. 


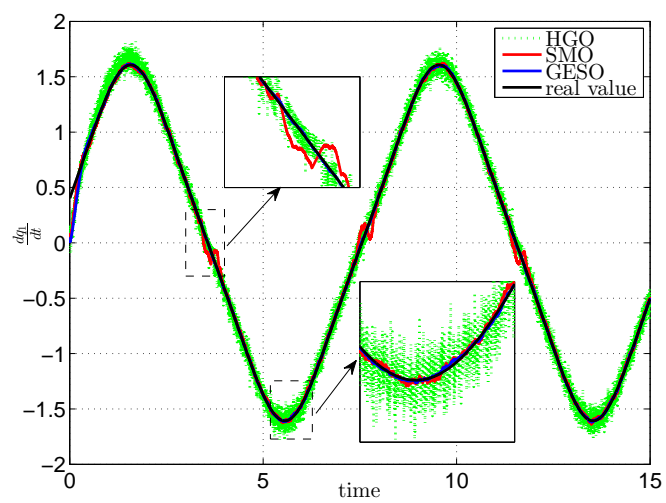

(a) Estimates of the velocity $\dot{q}_{1}$

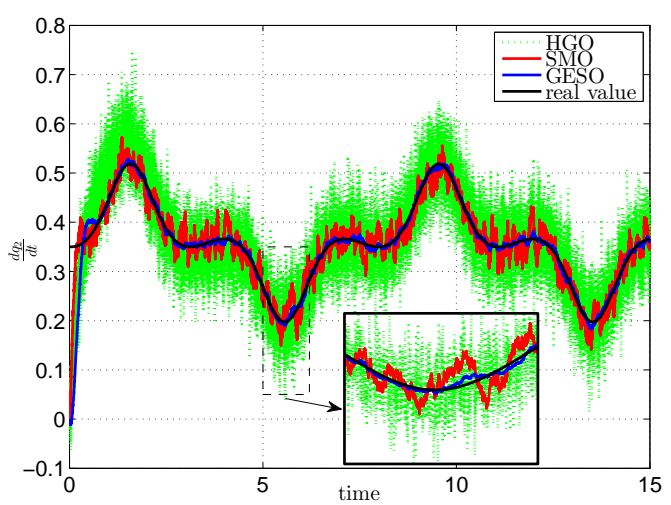

(b) Estimates of the velocity $\dot{q}_{2}$

Figure 3. Estimates of the velocities $\dot{q}_{1}$ and $\dot{q}_{2}$ with different observers

\subsection{Robotic leg}

This is an 3-dof, underactuation degree one, mechanical system depicted in Fig. 4. Its dynamics is described by (1) with $V(q)=0$,

$$
M(q)=\operatorname{diag}\left\{m_{1}, m_{1} q_{1}^{2}, m_{2}\right\}, G=\left[\begin{array}{rr}
1 & 0 \\
0 & -1 \\
0 & 1
\end{array}\right] .
$$

with $m_{1}>0, m_{2}>0, q_{1} \geq \epsilon>0$. As shown in (Venkatraman et al., 2010) the system is $\mathrm{PLvCC}$ with the matrix $\Psi(q)$ given as

$$
\Psi(q):=\left[\begin{array}{ccc}
\sin \left(q_{2}\right) & \sin \left(q_{2}\right) & 0 \\
\frac{1}{q_{1}} \cos \left(q_{2}\right)+\kappa & \frac{1}{q_{1}} \cos \left(q_{2}\right) & 0 \\
0 & 0 & 1
\end{array}\right], \kappa \neq 0,
$$

which is well-defined and full-rank for all $q_{2} \neq i \pi, i \in \mathbb{Z}_{+}$. This yields

$$
\mathcal{M}(q)=M(q)^{-1} \Psi^{-\top}(q)=\left[\begin{array}{ccc}
-\frac{\cot \left(q_{2}\right)}{\kappa m_{1} q_{1}} & \frac{\cos \left(q_{2}\right)+\kappa q_{1}}{\kappa m_{1} \sin (q 2) q_{1}} & 0 \\
\frac{1}{\kappa m_{1} q_{1}^{2}} & -\frac{1}{\kappa m_{1} q_{1}^{2}} & 0 \\
0 & 0 & \frac{1}{m_{2}}
\end{array}\right] .
$$

As in the previous example we compared in simulations the behaviour of the GESO (11) and the HGO and the SMO proposed in (Khalil \& Praly, 2014) and (Cruz-Zavala et al., 2010), respectively, which require the Coriolis matrix $C: \mathbb{R}^{3} \times \mathbb{R}^{3} \rightarrow \mathbb{R}^{3 \times 3}$ given by

$$
C(q, \dot{q})=\left[\begin{array}{ccc}
0 & -m_{1} q_{1} \dot{q}_{2} & 0 \\
m_{1} q_{1} \dot{q}_{2} & m_{1} q_{1} \dot{q}_{1} & 0 \\
0 & 0 & 0
\end{array}\right]
$$




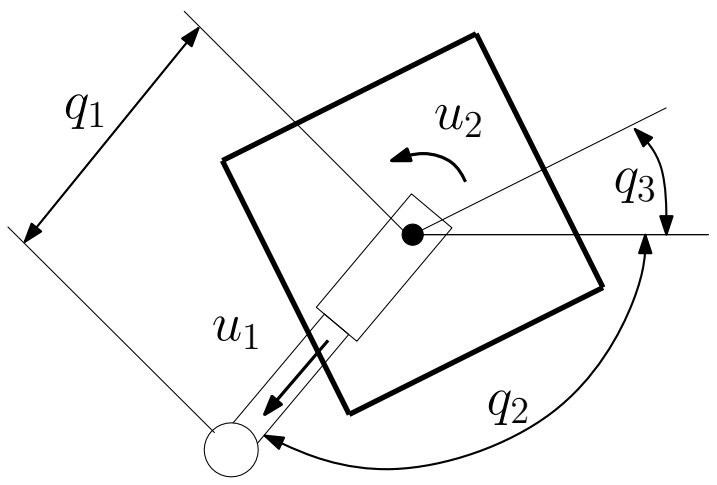

Figure 4. Robotic-leg system

The HGO is similar to (21), but now there are 3 -dimensional vectors

$$
\begin{aligned}
& \dot{\hat{x}}_{1}=\hat{x}_{2}+H_{1} \tilde{x}_{1}, \\
& \dot{\hat{x}}_{2}=M^{-1}\left(x_{1}\right)\left(G u-C\left(x_{1}, \hat{x}_{2}\right) \hat{x}_{2}\right)+H_{2} \tilde{x}_{1},
\end{aligned}
$$

where $H_{1}$ and $H_{2}$ are the constant matrices. The SMO also has the form (22) and is given by

$$
\begin{aligned}
& \dot{\hat{x}}_{1}=\hat{x}_{2}+k_{1} \Phi_{3}\left(\tilde{x}_{1}\right) \\
& \dot{\hat{x}}_{2}=M^{-1}\left(x_{1}\right)\left(G u-C\left(x_{1}, \hat{x}_{2}\right) \hat{x}_{2}\right)+k_{2} \Phi_{4}\left(\tilde{x}_{1}\right)
\end{aligned}
$$

where

$$
\begin{gathered}
\Phi_{3}\left(\tilde{x}_{1}\right)=\left[\begin{array}{l}
\mu_{1_{1}}\left|\tilde{x}_{1_{1}}\right|^{\frac{1}{2}} \operatorname{sign}\left(\tilde{x}_{1_{1}}\right)+\mu_{2_{1}}\left|\tilde{x}_{1_{1}}\right|^{\frac{3}{2}} \operatorname{sign}\left(\tilde{x}_{1_{1}}\right) \\
\mu_{1_{2}}\left|\tilde{x}_{1_{2}}\right|^{\frac{1}{2}} \operatorname{sign}\left(\tilde{x}_{1_{2}}\right)+\mu_{2_{2}}\left|\tilde{x}_{1_{2}}\right|^{\frac{3}{2}} \operatorname{sign}\left(\tilde{x}_{1_{2}}\right) \\
\mu_{1_{3}}\left|\tilde{x}_{1_{3}}\right|^{\frac{1}{2}} \operatorname{sign}\left(\tilde{x}_{1_{3}}\right)+\mu_{2_{3}}\left|\tilde{x}_{1_{3}}\right|^{\frac{3}{2}} \operatorname{sign}\left(\tilde{x}_{1_{3}}\right)
\end{array}\right], \\
\Phi_{4}\left(\tilde{x}_{1}\right)=\left[\begin{array}{l}
\frac{\mu_{1_{1}}^{2}}{2} \operatorname{sign}\left(\tilde{x}_{1_{1}}\right)+2 \mu_{2_{1}} \mu_{1_{1}} \tilde{x}_{1_{1}}+\frac{3}{2} \mu_{2_{1}}^{2}\left|\tilde{x}_{1_{1}}\right|^{2} \operatorname{sign}\left(x_{1_{1}}\right) \\
\frac{\mu_{1_{2}}^{2}}{2} \operatorname{sign}\left(\tilde{x}_{1_{2}}\right)+2 \mu_{2_{2}} \mu_{1_{2}} \tilde{x}_{1_{2}}+\frac{3}{2} \mu_{2_{1}}^{2}\left|\tilde{x}_{1_{2}}\right|^{2} \operatorname{sign}\left(x_{1_{2}}\right) \\
\frac{\mu_{1_{3}}^{2}}{2} \operatorname{sign}\left(\tilde{x}_{1_{3}}\right)+2 \mu_{2_{3}} \mu_{1_{3}} \tilde{x}_{1_{3}}+\frac{3}{2} \mu_{2_{1}}^{2}\left|\tilde{x}_{1_{3}}\right|^{2} \operatorname{sign}\left(x_{1_{3}}\right)
\end{array}\right],
\end{gathered}
$$

with free positive constant parameters $\mu_{i j}$ for $i, j=\{1,2,3\}$.

The system parameters were taken as $m_{1}=4.5, m_{2}=1.7$ and the change of coordinates gain set to $\kappa=5$. The simulation scenario is $u_{1}(t)=0.0535 \cos (10 t)$ and $u_{2}(t)=0.067 \sin (10 t), q(0)=[2.2,1.8,0.4]^{\top}$ and $\dot{q}(0)=p(0)=[0,0,0]^{\top}$ and initial conditions for the three observers $\hat{q}(0)=\left[\begin{array}{lll}0.1, & 0.2, & 0.4\end{array}\right]$ and $\hat{\dot{q}}(0)=\hat{p}(0)=\left[\begin{array}{lll}0, & 0,0\end{array}\right]^{\top}$. The SMO and HGO have been designed and tuned following the procedure suggested in (Cruz-Zavala et al., 2010) and (Khalil \& Praly, 2014), respectively, and are given as $H_{1}=\operatorname{diag}\{1.2,2,2\}, H_{2}=\operatorname{diag}\{3.9,2.5,1.3\}$ and $k_{1}=\operatorname{diag}\{1,0.52,0.71\}$, $k_{2}=\operatorname{diag}\{1,1.2,1.17\} ;$ and $\mu_{1 j}:=1.86$ and $\mu_{2 j}:=0.303$.

We have chosen the gains of the GESO as $L=\operatorname{diag}\{0.61,0.9,1.9\}$ and $\Gamma=$ $\operatorname{diag}\{1.74,4.9,0.71\}$ to approximately match the convergence rate of the other observers. 


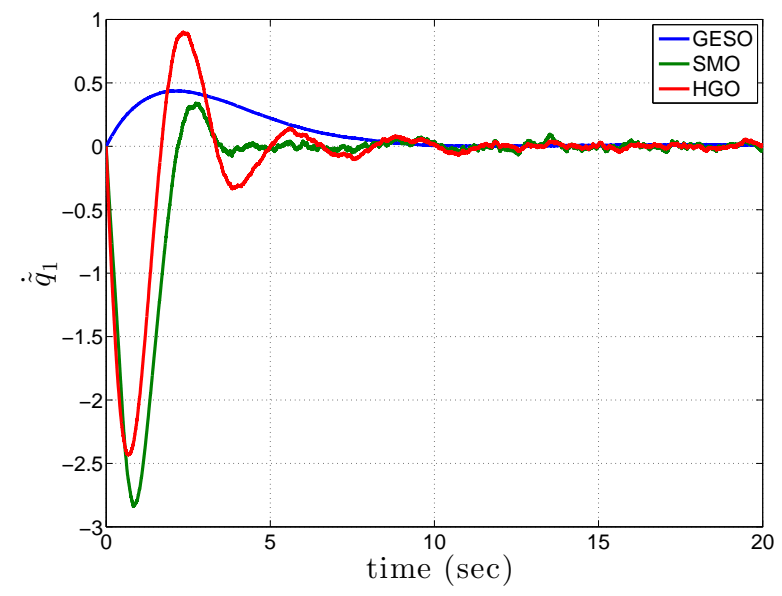

Figure 5. Transient behavior of $\dot{\tilde{q}}_{1}(t)$

Table 2. Numerical comparison of the observers.

\begin{tabular}{ccccccc}
\hline & \multicolumn{2}{c}{$\dot{q}_{1}$} & \multicolumn{2}{c}{$\dot{q}_{2}$} & \multicolumn{2}{c}{$\dot{q}_{3}$} \\
\cline { 2 - 7 } & $M E_{1} \cdot 10^{2}$ & $M S E_{1} \cdot 10^{4}$ & $M E_{2} \cdot 10^{2}$ & $M S E_{2} \cdot 10^{4}$ & $M E_{3} \cdot 10^{2}$ & $M S E_{3} \cdot 10^{4}$ \\
\hline HGO & 2.04 & 7.56 & 1.04 & 1.79 & 0.52 & 0.47 \\
\hline SMO & 2.14 & 7.25 & 2.20 & 7.76 & 0.86 & 0.98 \\
\hline GESO & 1.00 & 1.80 & 0.14 & 0.06 & 0.10 & 0.02 \\
\hline
\end{tabular}

In the ideal case when the position signals $q$ are measured without distortion all the observers have a good performance. Instead, we adopted the following realistic simulation scenario, which is similar to the scenario used in Subsection 5.1:

- The signals are affected by an additive normally distributed (Gaussian) random measurement noise with zero mean and variance of 0.1 .

- The noisy measurements are sampled with an analogue-to-digital converter with the quantization intervals $\frac{1}{500}$ for $q_{1}$ and $\frac{2 \pi}{256}$ for $q_{2}$ and $q_{3}$.

- The sampling frequency is fixed as $1 \mathrm{kHz}$.

The transient behaviors of the error signals $\dot{\tilde{q}}$ for the three observers are shown in Fig.5-7. As seen from the figures, the GESO has a somehow slower convergence rate for the first coordinate, but with a much smaller overshoot. On the other hand, it significantly outperforms the SMO and HGO for the other two coordinates. The excellent behaviour of the third speed observation error of the GESO stems from the fact that its dynamics is described by the linear homogeneous equation

$$
\ddot{\tilde{q}}_{3}+L_{3} \dot{\tilde{q}}_{3}+\frac{\Gamma_{3}}{m_{2}^{2}} \tilde{q}_{3}=0
$$

Making use of the metrics (23) a numerical comparison of the observers is presented in Table 2 where - as in the previous example - the transients have been omitted. It can be seen that in all coordinates and metrics the proposed GESO largely outperforms the HGO and the SMO. 


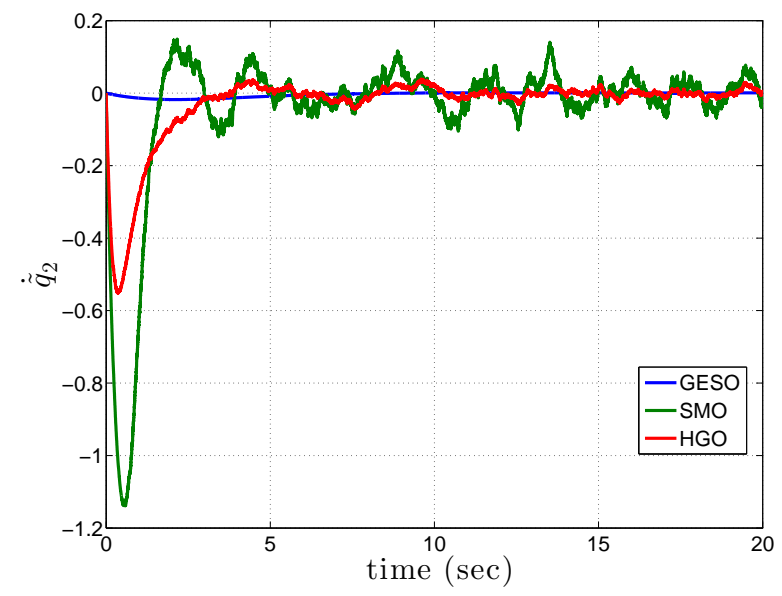

Figure 6. Transient behavior of $\dot{\tilde{q}}_{2}(t)$

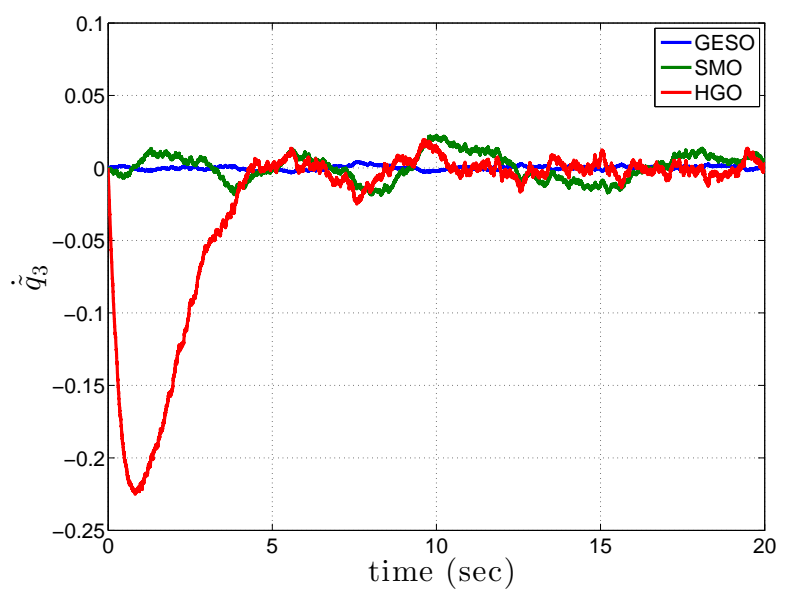

Figure 7. Transient behavior of $\dot{\tilde{q}}_{3}(t)$

\section{Experimental Results}

\subsection{Experimental setup description}

For experimental studies, we use the cart-pendulum equipment shown in Fig. 8 that is assembled from off-the-shelf components and is located at LORIA laboratory, France. In contrast with the normalized model (20) we consider the full model

$$
\begin{gathered}
M(q)=\left[\begin{array}{cc}
J_{f} & l_{m} m_{p} \cos \left(q_{1}\right) \\
l_{m} m_{p} \cos \left(q_{1}\right) & M_{c}+m_{p}
\end{array}\right], \\
V(q)=l_{m} m_{p} \cos \left(q_{1}\right), G=\left[\begin{array}{l}
0 \\
1
\end{array}\right], \\
C(q, \dot{q})=\left[\begin{array}{cc}
0 & 0 \\
-l_{m} m_{p} \dot{q}_{1} \sin \left(q_{1}\right) & 0
\end{array}\right] .
\end{gathered}
$$




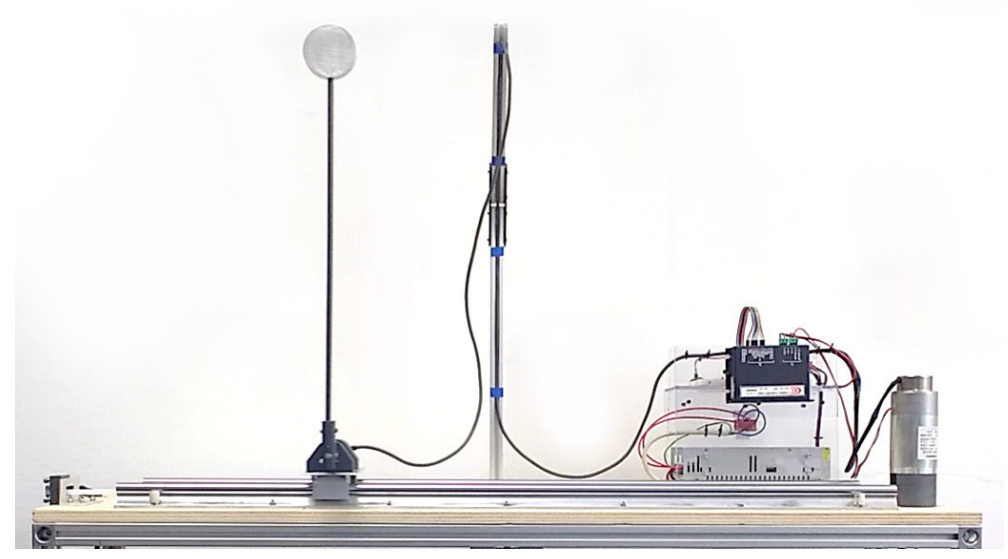

Figure 8. Photo of the cart-pendulum experimental setup

Table 3. Parameters of the experimental setup.

\begin{tabular}{ccc} 
Description & Symbol & Value \\
\hline Mass of the cart, $\mathrm{kg}$ & $M_{c}$ & 0.85 \\
\hline Mass of the pendulum, $\mathrm{kg}$ & $m_{p}$ & 0.30 \\
\hline Distance to the center of mass of the pendulum, $\mathrm{m}$ & $l_{m}$ & 0.35 \\
\hline Moment of inertia of the pendulum, $\mathrm{kg} \cdot \mathrm{m}^{2}$ & $J_{f}$ & $4.84 \cdot 10^{-2}$ \\
\hline Gain between the motor current and the force acting on the cart, N/A & - & 9.27 \\
\hline Coulomb friction of the cart for positive velocities, $\mathrm{N}$ & - & 3.98 \\
\hline Coulomb friction of the cart for negative velocities, $\mathrm{N}$ & - & -2.87
\end{tabular}

where, to recall, $q=\left[q_{1}, q_{2}\right]^{\top}, q_{1}$ is the pendulum's angular position with zero at the upright position and $q_{2}$ is the cart's position. The cart is driven by a $24 \mathrm{~V}$ DC Servo Motor (Shinko Electric) via a Copley Servo Amplifier (Model 403) having an internal current loop. The signals are processed by an Arduino board (Nano v3.0), velocity observers and control signals are computed by the same board. Other parameters of the experimental setup are given in Table 3; these parameters were identified via a set of open-loop experiments using nonlinear grey-box prediction error minimization methods, see (Ljung (1999)). The friction of the cart is modeled as an asymmetric Coulomb friction, and friction of the pendulum is neglected. It worth noting that, due to the non-negligible mass of the rod, the moment of inertia of the pendulum is not equal to the moment of inertia of an ideal pendulum, namely $m_{p} l_{m}^{2}$.

The setup is equipped with optical encoders providing precise measurements of cart and pendulum positions. Further, we consider two scenarios of available measurements: high-precision measurements (HPM) and low-precision measurements (LPM). For high-precision measurements we use the best measurements available in the setup with a high sampling frequency. For low-precision measurements we reduce the sampling frequency and mimic position sensors with larger quantization levels. These two scenarios allow us to study and illustrate performance and applicability of various approaches for the varied quality of sensors. Details on these scenarios are given in Table 4. In all scenarios we use Euler forward integration method and single-precision float-point arithmetics. 
Table 4. Two scenrios of avaliable measurements.

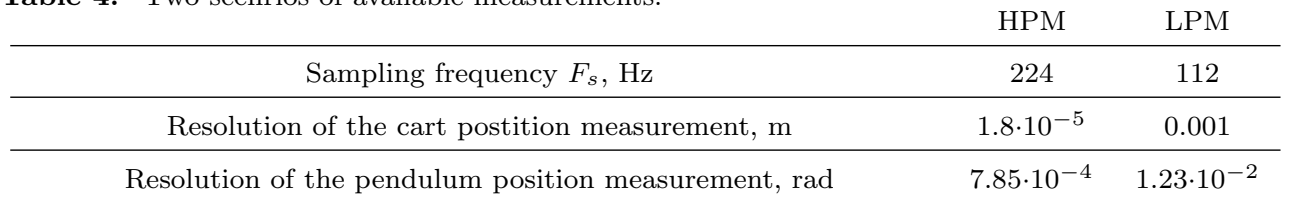

\subsection{Open-loop comparison}

As in simulations, we compare three observers: the GESO given by (11), the SMO given by (22), and an extended version of the HGO (21) further denoted as EHGO and given by

$$
\begin{aligned}
& \dot{\hat{x}}_{1}=\hat{x}_{2}+\left(\frac{h_{1}}{\varepsilon_{h}}\right) \tilde{x}_{1}, \\
& \dot{\hat{x}}_{2}=\sigma_{x}+M^{-1}\left(x_{1}\right)\left[G u-\nabla V\left(x_{1}\right)-C\left(x_{1}, \hat{x}_{2}\right) \hat{x}_{2}\right]+\left(\frac{h_{2}}{\varepsilon_{h}^{2}}\right) \tilde{x}_{1}, \\
& \dot{\sigma}_{x}=\left(\frac{h_{3}}{\varepsilon_{h}^{3}}\right) \tilde{x}_{1} .
\end{aligned}
$$

Extended observer (25) contains additional states $\sigma_{x}$ which denote estimates of model uncertainties - making it coincide with the observer in (Lee et al. (2015)).

In the considered open-loop experiment the motor is excited with an input current signal consisting of two parts, where the first part is a chirp signal having the magnitude $2.5 \mathrm{~A}$ and the frequency varying from 2 to $9 \mathrm{~Hz}$ in 5 seconds. Next, after a rest interval of 1 second, the input current switches to a square wave having the magnitude $0.8 \mathrm{~A}$ and the frequency $2 \mathrm{~Hz}$; the total duration of the experiment is 11 seconds. As a baseline for velocity estimation we use an optimal off-line fitting of the measured position with smoothing splines taking into account quantization level and minimizing the integral of the squared acceleration, see (Vázquez, Aranovskiy, Freidovich, and Fridman (2016)) for more details. Trajectories $q_{1}(t)$ and $q_{2}(t)$ measured in the considered experiment and (off-line estimated) velocities $\dot{q}_{1}(t)$ and $\dot{q}_{2}(t)$ are given if Fig. 9. It also worth noting that this open-loop experiment was not used for model parameters identification.

We use the trajectories measured under the high-precision measurements scenario to tune the observers. For the GESO the tuning is done in order to obtain smooth velocity estimates yielding $L=\operatorname{diag}\{50,80\}$ and $\Gamma=\operatorname{diag}\{400,150\}$. For the SMO and EHGO we have the following options:

- Following Remark 1 in (Davila et al., 2005), for the SMO we choose $\mu_{1}$ and $\mu_{2}$ as the double maximal accelerations observed for the system, that is $\mu_{1}=2 \cdot 39=78$ and $\mu_{2}=2 \cdot 14=28$. This tuning of the SMO is further denoted as ConVentional, SMO-CV.

- Following section 4 in (Lee et al., 2015), for the EHGO we choose $h_{1}=5, h_{2}=5$ and $h_{3}=4$; note that the parameters of the equipment used in (Lee et al., 2015) are comparable to the parameters of the setup used in this paper. Unfortunately, the value $\varepsilon_{h}=0.002$ proposed in (Lee et al., 2015) is too aggressive for our setup and leads to (numerical) instability under forward Euler integration with the sampling frequency specified for the high-precision measurements scenario, see Table 4 . To obtain a working solution we had to increase $\varepsilon_{h}$, i.e. to decrease the 


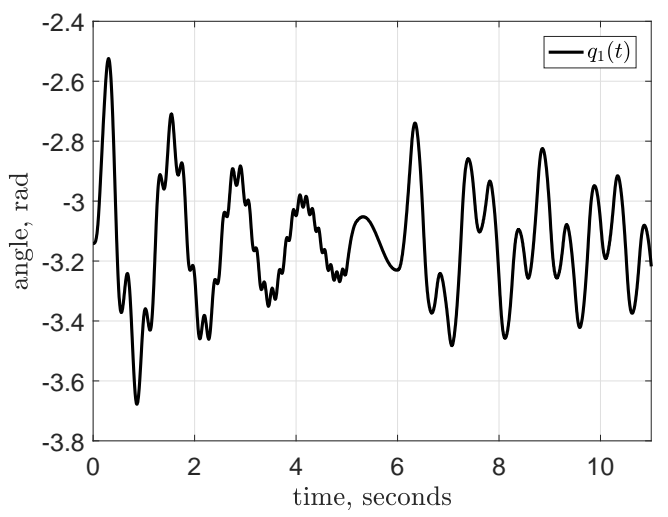

(a) Position of the pendulum $q_{1}(t)$

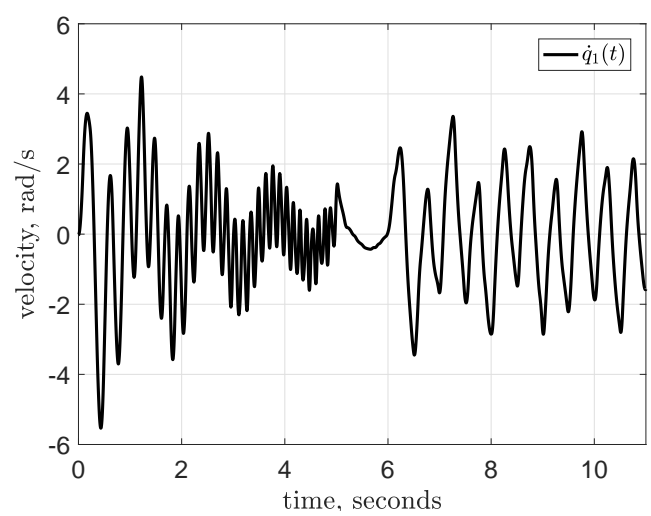

(c) Velocity of the pendulum $\dot{q}_{1}(t)$

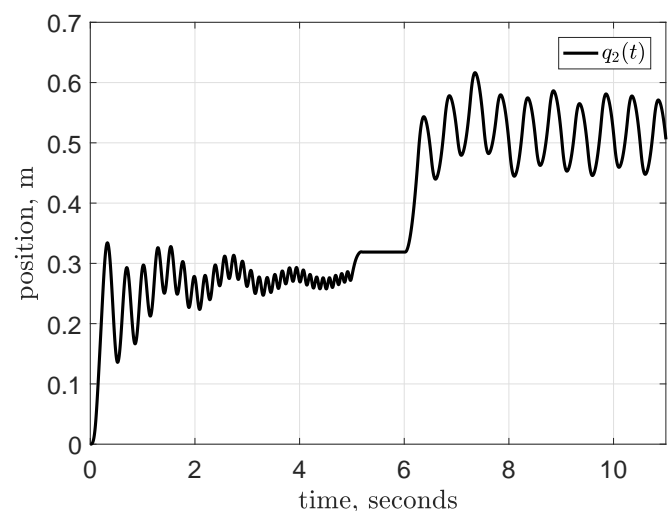

(b) Position of the cart $q_{2}(t)$

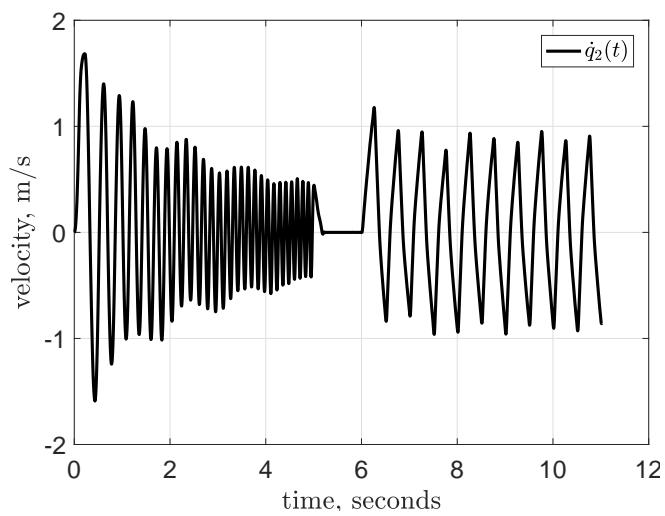

(d) Velocity of the cart $\dot{q}_{2}(t)$

Figure 9. Trajectories of the system in the open-loop experiment

observer gains, up to $\varepsilon_{h}=0.009$. This tuning of the EHGO is further denoted as ConVentional, EHGO-CV.

- To be able to present a fair comparison of observers we also tune the SMO and the EHGO in such a way that all observers have (approximately) equal performance index defined as

$$
J_{\text {perf }}=M S E_{1}+M S E_{2},
$$

where $M S E$ is the mean square error metric defined in (23). For the SMO this goal is achieved for $\mu_{1}=29$ and $\mu_{2}=10$; this baseline tuning is further denoted as SMO-BL. For the EHGO the goal is achieved for the same $h_{1}, h_{2}$ and $h_{3}$ as in EHGO-CV with $\varepsilon_{h}=0.0126$; this baseline tuning is further denoted as EHGO-BL.

We are now in a position to present observers comparison for the considered openloop experiment. The metrics $M E$ and $M S E$ (computed as (23) with respect to the off-line estimated velocity) are given in Table 5 for all observers and both highprecision and low-precision measurements scenarios, and illustrations of the outputs of the baseline-tuned observers for the low-precision measurements scenario are given in Fig. 10 for the pendulum and in Fig. 11 for the cart. The obtained results are discussed in Subsection 6.4. 
Table 5. Comparison of the observers in the open-loop experiment

\begin{tabular}{ccccc}
\hline & & High-precision measurements & \\
& $M E_{1} \times 10^{2}$ & $M S E_{1} \times 10^{2}$ & $M E_{2} \times 10^{2}$ & $M S E_{2} \times 10^{2}$ \\
\hline GESO & 13.9 & 356.4 & 5.2 & 38.6 \\
\hline SMO-BL & 15.2 & 376.4 & 4.3 & 28.9 \\
\hline SMO-CV & 28.8 & 359.2 & 4.0 & 30.1 \\
\hline EHGO-CV & 45.4 & 3258.4 & 9.3 & 130.8 \\
\hline GHG-BL & 14.4 & Low-precision measurements & \\
\hline SMO-BL & 18.4 & 403.0 & 5.1 & 37.8 \\
\hline EHGO-BL & 75.7 & 518.0 & 5.5 & 48.4 \\
\hline SMO-CV & 34.3 & 1834.0 & 24.2 & 950.3 \\
\hline EHGO-CV & 1191.2 & $2643.10^{3}$ & 290.0 & $205 \cdot 10^{3}$ \\
\hline
\end{tabular}

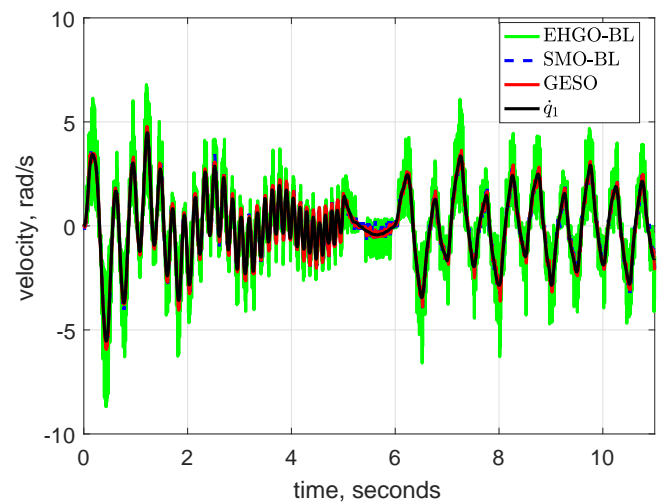

(a) Velocity estimates, full time scale

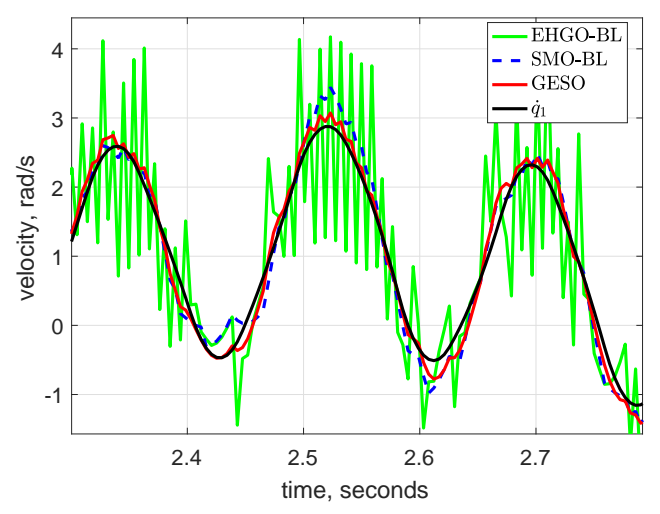

(b) Velocity estimates, zoomed

Figure 10. Velocity of the pendulum and the observers' outputs for the low-precision measurements scenario.

\subsection{Closed-loop comparison}

For the closed-loop experiment we consider the problem where the cart is supposed to track a given reference while keeping the pendulum in the upright position. The reference is chosen as a saw-like signal with magnitude $0.2 \mathrm{~m}$. The control law is derived as an LQR computed for the linear model corresponding to the linearization at the upright position of the system and is given by

$$
u(r, q, \dot{q})=21\left(r-q_{2}\right)+23\left(\dot{r}-\hat{\dot{q}}_{2}\right)-104 q_{1}-20 \hat{\dot{q}}_{1},
$$

where $r(t)$ is the reference signal for the cart, and $\hat{\dot{q}}$ is an estimate of $\dot{q}$.

Unfortunately, the EHGO-BL used in feedback was not able to stabilize the system; to obtain a working solution we had to increase $\varepsilon_{h}$ form 0.0126 (EHGO-BL) to 0.025 . Recalling (25), it implies that the gain for $\dot{\sigma}_{x}$ was, particularly, decreased by a factor of (approximately) 8. The EHGO with this set of parameters is not further evaluated.

The GESO and SMO-BL are both able to stabilize the system using the highprecision measurements. For the low-precision measurements case the GESO still stabilizes the system, while the SMO-BL keeps the pendulum upright but does not track 


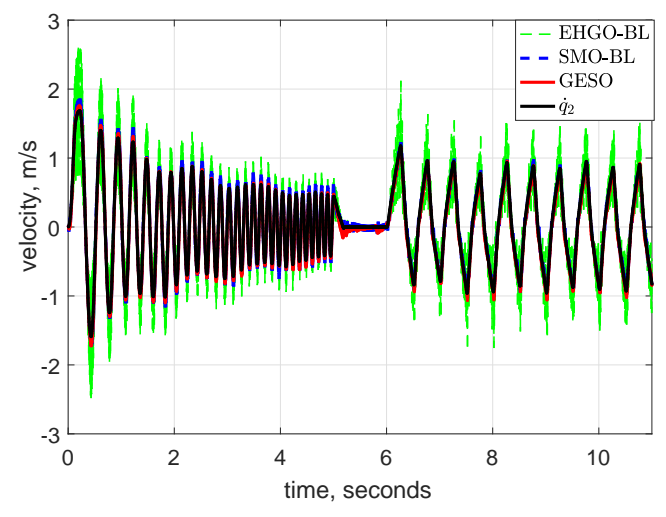

(a) Velocity estimates, full time scale

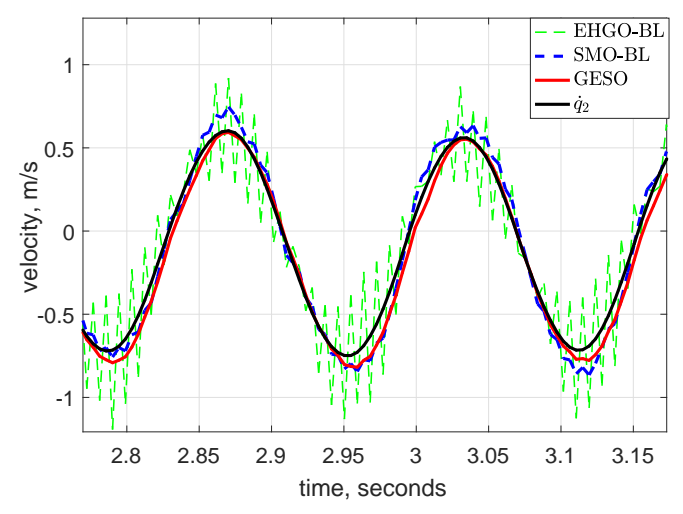

(b) Velocity estimates, zoomed

Figure 11. Velocity of the cart and the observers' outputs for the low-precision measurements scenario.

Table 6. Comparison of the observers in the open-loop experiment

\begin{tabular}{ccc}
\hline & \multicolumn{2}{c}{ High-precision measurements } \\
& $\frac{1}{N} \sum_{i=1}^{N}\left|q_{1}(i)\right| \times 10^{2}$ & $\frac{1}{N} \sum_{i=1}^{N} q_{1}^{2}(i) \times 10^{4}$ \\
\hline GESO & 0.59 & 1.08 \\
\hline SMO-BL & 0.93 & 1.57 \\
\hline & Low-precision measurements \\
\cline { 2 - 3 } GESO & 1.03 & 2.42 \\
\hline
\end{tabular}

the reference and does not keep the cart in the admissible region $\left|q_{2}\right| \leq 0.3 \mathrm{~m}$. Table 6 presents mean absolute tracking error and mean squared tracking error for the pendulum, where it can be seen that for the same stabilizing LQR controller the GESO in feedback provides better pendulum stabilization. Trajectories of the closed-loop system for high-precision measurements with the GESO and SMO-BL are given in Fig. 12 and Fig. 13, respectively. Computational time for each observer is given in Table 7 .

\subsection{Discussion}

The previous study leads to the following conclusions.

C1 For the high-precision measurements scenario, i.e. for a fast sampling frequency and precise position sensors, all observers can be tuned to have similar performances, see the upper part of Table 5. However, if the same observers are used with the low-precision measurements, then the performance of the EHGOBL and SMO-BL significantly deteriorates, while the performance of the GESO changes slightly, see the lower part of Table 5. It illustrates that the GESO is more robust with respect to sensor noises and imperfections.

C2 Recall that the SMO-BL and EHGO-BL are tuned to have competitive (with the

Table 7. Computational time for one Forward-Euler integration step, Arduino Nano v3.0 board

\begin{tabular}{cccc}
\hline & GESO & SMO-BL & EHGO-BL \\
\hline Computational time, $\mu \mathrm{s}$ & 466 & 551 & 472
\end{tabular}




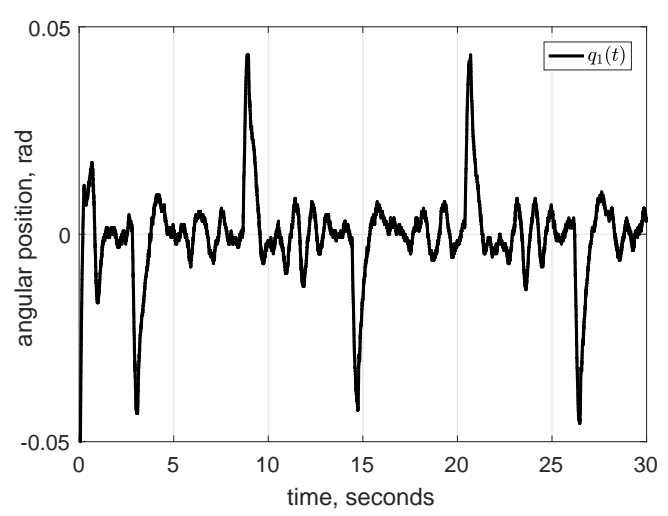

(a) Pendulum position $q_{1}(t)$

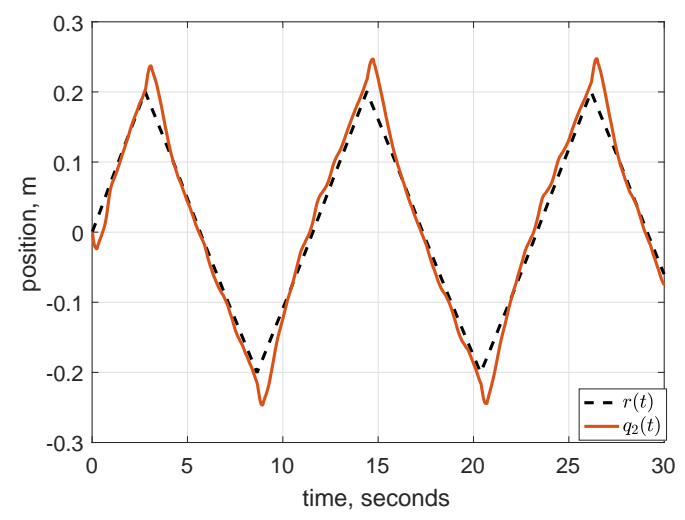

(b) Reference $r(t)$ and cart position $q_{2}(t)$

Figure 12. Closed-loop reference tracking with the GESO, high-precision measurements.

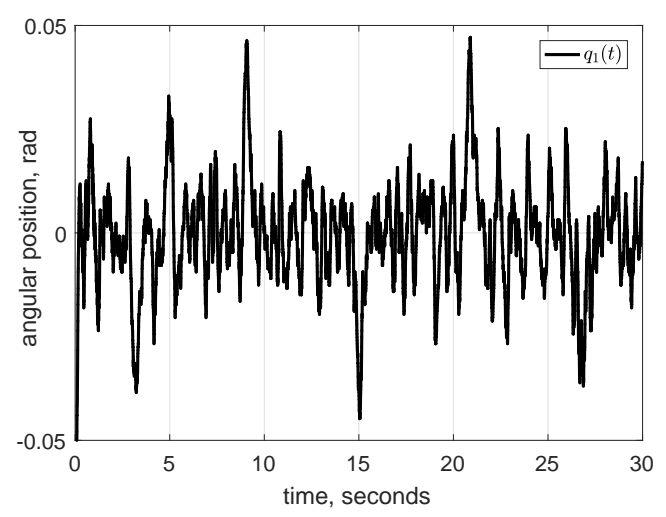

(a) Pendulum position $q_{1}(t)$

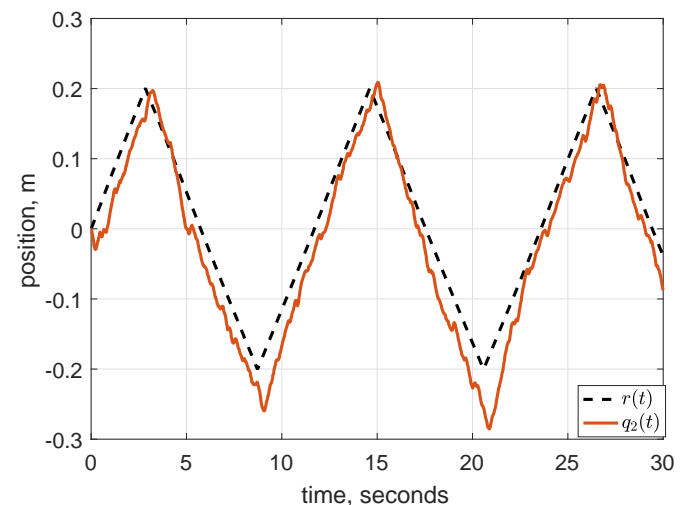

(b) Reference $r(t)$ and cart position $q_{2}(t)$

Figure 13. Closed-loop reference tracking with the SMO-BL, high-precision measurements.

GESO) performance. In order to achieve this goal the gains of the SMO and EHGO are notably decreased, while the performance of these observers under the convenient tuning recommendations is significantly worse, see the SMO-CV and EHGO-CV lines in Table 5. The drawback of decreasing these gains is that the stability and applicability of the SMO and EHGO is analytically proven only for sufficiently high gains, see (Davila et al., 2005) and (Lee et al., 2015). In other words, for the SMO and EHGO decreasing the gains in order to improve performance can compromise the convergence property of these observers. It worth noting that for the GESO the global exponential stability is ensured for all positive gains $L$ and $\Gamma$.

C3 Despite the fact that the GESO is based on model knowledge, it is shown to be sufficiently robust with respect to inevitable model uncertainties. This fact is illustrated by the successful implementation of the GESO in the closed-loop pendulum stabilization system.

C4 The derivation of the proposed GESO involves some coordinates transformation and may seem complicated. However, the resulting observer equations are not more complex than the ones of the SMO and EHGO. This observation is also supported by the fact that computational time for all three observers is almost equal, see Table 7 . 


\section{Concluding Remarks}

It has been shown in the paper that the problem of speed (or momenta) observation of PLvCC mechanical systems admits a very simple - even trivial-Luenberger-like solution. In spite of its remarkable simplicity this fact does not seem to have been reported in the literature, where only far more complicated observers are available. The observer ensures the very strong property of GES of the error system without any excitation requirement. The only assumption on the input is the usual boundedness of trajectories condition. The GES nature of the proposed observer makes it a suitable candidate to - combined with a full-state GAS controller à la (Venkatraman et al., 2010) - yield a GAS solution to the position feedback stabilization problem, but the details are omitted for brevity.

It should be noted that, although the assumption of PLvCC may seem restrictive, the class contains a very long list of benchmark examples, including the pendulum on a cart, the mass and beam system, the spherical pendulum on a puck, the 3-link underactuated planar manipulator and the planar redundant manipulator with one elastic degree of freedom. In a recent paper (Chang, Song, \& Kim, 2016) this class has been enlarged adding to the change of coordinates a position feedback term. New sufficient conditions for quasilinearizability are given and are shown to be satisfied by the Acrobot example. It is interesting to see how the presence of this new term affects the observation problem.

Another contribution of the paper is to exhibit, via realistic simulations and very detailed experiments, the high sensitivity to noise of HGO and SMO in mechanical systems. It should be underscored that a lot of effort is under way to palliate this problem for high gain observers, in particular, to reduce the peaking phenomenon when they are applied to high order systems, e.g., (D. Astolfi \& Marconi, 2015; Teel, 2016). However, these modifications have no impact on second order mechanical equations.

At a more philosophical level, we quote below Slavoj Zizek (Žižek, 1989) and ask ourselves if all these fixes are merely a Ptolemization of an intrinsically fragile designthat contradicts a basic premise of control theory to avoid high gains in feedback systems.

"When a discipline is in crisis, attempts are made to change or supplement its theses within the terms of its basic framework - a procedure one might call 'Ptolemization' (since when data poured in which clashed with Ptolemy's earth-centred astronomy, his partisans introduced additional complications to account for the anomalies). But the true 'Copernican' revolution takes place when, instead of just adding complications and changing minor premises, the basic framework itself undergoes a transformation. So, when we are dealing with a self-professed 'scientific revolution', the question to ask is always: is this truly a Copernican revolution, or merely a Ptolemization of the old paradigm?"

S. Zizek

\section{Acknowledgment}

The authors are grateful to Frederic Mazenc for help in the proof of Proposition 4.1 and Elena Panteley for some clarifications on PE and stability of the system (13) that significantly influenced the paper. 


\section{Funding}

This article is supported by Government of Russian Federation (grant 074-U01, GOSZADANIE 8.8885.2017), the Ministry of Education and Science of Russian Federation (project 14.Z50.31.0031).

\section{References}

Anderson, B. D. O., Bitmead, R. R., Johnson, C. R., Jr., Kokotovic, P. V., Kosut, R. L., Mareels, I. M., ... Riedle, B. (1986). Stability of adaptive systems: Passivity and averaging analysis. Cambridge, MA, USA: MIT Press.

Astolfi, A., Karagiannis, D., \& Ortega, R. (2007). Nonlinear and adaptive control with applications. London: Springer Science \& Business Media.

Astolfi, D., \& Marconi, L. (2015, Nov). A high-gain nonlinear observer with limited gain power. IEEE Transactions on Automatic Control, 60(11), 3059-3064.

Barabanov, N., \& Ortega, R. (2017, January). On global asymptotic stability of $\dot{x}=\phi(t) \phi^{\top}(t) x$ with $\phi(t)$ bounded and not persistently exciting. LSS Int. Report.

Bedrossian, N., \& Spong, M. W. (1995). Feedback linearization of robot manipulators and riemannian curvature. Journal of Robotic Systems, 12, 541-552.

Chang, D. E., \& McLenaghan, R. G. (2013, April). Geometric criteria for the quasilinearization of the equations of motion of mechanical systems. IEEE Transactions on Automatic Control, 58(4), 1046-1050.

Chang, D. E., Song, S., \& Kim, J. K. (2016). A sufficient condition for the feedback quasilinearization of control mechanical systems. Journal of Electrical Engineering and Technology, $11(3), 741-745$.

Cruz-Zavala, E., Moreno, J. A., \& Fridman, L. (2010, June). Uniform second-order sliding mode observer for mechanical systems. In 2010 11th international workshop on variable structure systems (vss) (pp. 14-19).

Davila, J., Fridman, L., \& Levant, A. (2005, Nov). Second-order sliding-mode observer for mechanical systems. IEEE Transactions on Automatic Control, 50(11), 1785-1789.

Esfandiari, F., \& Khalil, H. K. (1992). Output feedback stabilization of fully linearizable systems. International Journal of Control, 56 (5), 1007-1037.

Ioannou, P. A., \& Sun, J. (2012). Robust adaptive control. Courier Corporation.

Khalil, H. K. (2002). Nonlinear systems. New Jersey: Prentice Hall.

Khalil, H. K., \& Praly, L. (2014). High-gain observers in nonlinear feedback control. International Journal of Robust and Nonlinear Control, 24(6), 993-1015.

Krener, A. J., \& Respondek, W. (1985). Nonlinear observers with linearizable error dynamics. SIAM Journal on Control and Optimization, 23(2), 197-216.

Lee, J., Mukherjee, R., \& Khalil, H. K. (2015). Output feedback stabilization of inverted pendulum on a cart in the presence of uncertainties. Automatica, 54, 146-157.

Ljung, L. (1999). System identification: theory for the user (Second ed.). Prentice Hall PTR.

Loria, A. (2004). Explicit convergence rates for mrac-type systems. Automatica, 40(8), 1465 -1468 .

Maghenem, M., \& Loria, A. (2016, December). Lyapunov functions for persistently-excited cascaded time-varying systems: Application to consensus. LSS Int. Report.

Malisoff, M., \& Mazenc, F. (2009). Constructions of strict lyapunov functions. Springer Science \& Business Media.

Marino, R., \& Tomei, P. (1996). Nonlinear control design: geometric, adaptive and robust. Prentice Hall International (UK) Ltd.

Morgan, A. P., \& Narendra, K. S. (1977). On the uniform asymptotic stability of certain linear nonautonomous differential equations. SIAM Journal on Control and Optimization, $15(1), 5-24$. 
Panteley, E., \& Loria, A. (1998). On global uniform asymptotic stability of nonlinear timevarying systems in cascade. Systems \&3 Control Letters, 33(2), 131 - 138.

Romero, J. G., \& Ortega, R. (2015). Two globally convergent adaptive speed observers for mechanical systems. Automatica, 60, $7-11$.

Sastry, S., \& Bodson, M. (2011). Adaptive control: stability, convergence and robustness. Courier Corporation.

Spivak, M. (1999). A comprehensive introduction to differential geometry. Cambridge, MA, USA: Perish, Inc.

Teel, A. R. (2016, July). Further variants of the astolfi/marconi high-gain observer. In 2016 american control conference (acc) (p. 993-998).

Vázquez, C., Aranovskiy, S., Freidovich, L., \& Fridman, L. (2016). Time-varying gain differentiator: A mobile hydraulic system case study. IEEE Transactions on Control Systems Technology, 24(5), 1740-1750.

Venkatraman, A., Ortega, R., Sarras, I., \& Van der Schaft, A. (2010). Speed observation and position feedback stabilization of partially linearizable mechanical systems. IEEE Transactions on Automatic Control, 55(5), 1059.

Žižek, S. (1989). The sublime object of ideology. London: Verso Eds. 